\section{PAPEL DO FISIOTERAPEUTA NO ENVELHECIMENTO ATIVO}

\section{ROLE OF THE PHYSIOTHERAPIST IN ACTIVE AGING}

Gabriela Carvalho Leão (Orcid: 0000-0001-5878-5726) ${ }^{1}$

Jhonatan Carlos Terencio Ribeiro (Orcid: 0000-0003-0648-0538)

Angelo Piva Biagini (Orcid: 0000-0003-3187-5830) ${ }^{2}$

Carlos Fernando Ronchi (Orcid: 0000-0002-5927-9320) ${ }^{1,2}$

Edneia de Oliveira Salum (Orcid: 0000-0003-2342-9400)

Marilita Falangola Accioly (Orcid: 0000-0002-9623-3145)1,3

Isabel Aparecida Porcatti de Walsh (Orcid: 0000-0002-2317-1326) ${ }^{1,3}$

\title{
RESUMO
}

A população brasileira encontra-se em um intenso processo de envelhecimento que, associado à transição epidemiológica, estimulou a criação de diversas políticas públicas em prol de melhorias na qualidade de vida dos idosos, visando um ambiente favorável para um envelhecimento ativo. Visto isso, e considerando o alto custo dos gastos em saúde da população idosa, faz-se necessário o estudo do processo de saúde e dos seus agentes promotores. A inatividade física desempenha um fator importante no desenvolvimento de doenças crônicas não transmissíveis, gerando agravos e levando o idoso a incapacidades. Nesse contexto, o fisioterapeuta atua como promotor de saúde, cabendo, assim, uma reflexão sobre a sua atuação no envelhecimento ativo. O envelhecimento ativo é influenciado pelas escolhas relacionadas com o estilo de vida saudável, bem como com os determinantes sociais. A Atenção Primária, por intermédio da adscrição dos idosos, visa criar vínculos entre os profissionais da saúde e a população com o intuito de estimular a corresponsabilização com saúde e, consequentemente, melhorar a qualidade de vida. Para a efetividade dessas ações, são imprescindíveis o conhecimento da população e suas demandas, dos dispositivos do território e o reconhecimento que isso difere nas regiões brasileiras. O fisioterapeuta dispõe de ferramentas que buscam minimizar os agravos, reduzir complicações e promover a saúde no envelhecimento. No entanto, ele ainda é visto como um profissional reabilitador, tanto pela comunidade quanto pelos outros profissionais de saúde, o que gera limitações no escopo de atuação, fazendo-se necessária a educação permanente desses para que possam considerá-lo como um apoiador da Atenção Básica.

Palavras-chave: Envelhecimento; Saúde do Idoso; Fisioterapia.

\section{Contato}

Isabel Aparecida Porcatti de Walsh

E-mail: isabelpwalsh@gmail.com

${ }^{1}$ Programa de Pós-Graduação em Fisioterapia, Universidade Federal do Triângulo Mineiro, Universidade Federal de Uberlândia, Minas Gerais, Brasil.

${ }^{2}$ Curso de Fisioterapia, Universidade Federal de Uberlândia, Minas Gerais, Brasil.

${ }^{3}$ Departamento de Fisioterapia Aplicada, Universidade Federal do Triângulo Mineiro, Minas Gerais, Brasil.

\section{ABSTRACT}

The Brazilian population is in an intense aging process that, when associated with the epidemiological transition, stimulated the creation of several public policies in favor of improvements in the quality of life of the elderly aiming at a favorable environment for an active aging. Given this scenario, and considering the high cost of health expenditures of the elderly population, it is necessary to study the health process and its promoters. Physical inactivity plays an important role in the development of chronic noncommunicable diseases causing injuries and leading the elderly to disability. In this context, the physiotherapist acts as a health promoter, thus reflecting on his role as a promoter of active aging. Active aging is influenced by choices related to a healthy lifestyle as well as social determinants. Primary Care, through the ascription of the elderly, aims to create links between health professionals and the population with the purpose of stimulating the co-responsibility of health and consequently improving the quality of life. For the effectiveness of these actions, it is necessary to know the population and its demands, the devices of the territory and the recognition that this differs throughout Brazilian regions. The physiotherapist has tools that seek to minimize injuries, reduce complications, and promote health in aging. However, the physiotherapist is still seen as a rehabilitation professional, both by the community and by other health professionals, which generates limitations in the scope of action making it necessary the permanent education of health professionals, so that they can consider the physiotherapist as professional supporter of Primary Care.

Keywords: Aging; Health of the elderly; Physical therapy specialty. 
POLÍTICAS PÚBLICAS E IDOSO

O Brasil vivência o processo de envelhecimento da população, resultado da redução da taxa de fecundidade, natalidade e mortalidade e do aumento da expectativa de vida dos brasileiros ${ }^{1}$. Em 2017, pessoas com 60 anos ou mais representavam 14,6\% da população brasileira ${ }^{2}$. Estimativas do IBGE apontam que esse valor deve chegar a 29\% em 2050, e que, até 2025, o País será o sexto país do mundo com maior número de idosos ${ }^{2,3}$.

Outro fenômeno que acontece em consonância com o envelhecimento populacional no Brasil é o processo de transição epidemiológica, caracterizado pela redução da prevalência de doenças infectocontagiosas, mas ainda presente, e aumento da prevalência de doenças crônicas não transmissíveis (DCNT), comumente encontradas nas faixas etárias mais elevadas ${ }^{4}$.

Os processos de transição epidemiológica e demográfica implicam desafios relacionados com a assistência à saúde, uma vez que parte considerável dos gastos públicos em saúde é direcionada à população idosa. Desse modo, nos próximos anos, espera-se maior investimento financeiro, visto que as internações hospitalares dos idosos são mais frequentes e o tempo de ocupação no leito é maior quando comparado a outras faixas etárias. Além disso, as doenças crônicas em idosos exigem acompanhamento constante, cuidados permanentes, exames periódicos e medicação contínua ${ }^{5}$.
Sabe-se ainda do impacto que as doenças crônicas trazem para o idoso, família e comunidade, já que estão diretamente relacionadas com a incapacidade funcional, refletindo na dependência do idoso, contribuindo para o aumento da vulnerabilidade, diminuição do bem-estar e da qualidade de vida dele ${ }^{6}$.

Estudos recentes têm mostrado que as doenças crônicas, bem como as incapacidades advindas do adoecimento, influenciam no envelhecimento ativo, uma vez que se relacionam com uma condição de saúde comprometida que resulta em menor qualidade de vida, mas que são evitáveis por meio das ações de prevenção ${ }^{7}$.

Nesse caso, as ações de prevenção proporcionam benefícios em qualquer nível de atenção e nas fases mais tardias da vida ${ }^{7}$, e o fisioterapeuta se mostra um profissional capacitado para auxiliar nesse processo, por ser habilitado a intervir na prevenção de doenças e seus agravos, na reabilitação e na educação e promoção à saúde nos diversos níveis de atenção, sendo a atenção primária a base prioritária em saúde pública ${ }^{8}$.

No Brasil, as políticas públicas englobam ações de melhorias em qualidade de vida dos idosos ${ }^{9}$. No entanto, o processo de implantação dessas ações, em todo o território brasileiro, é marcado pela dificuldade, principalmente no acompanhamento do rápido crescimento dessa população. Diante do exposto, cabe a reflexão sobre qual o papel do fisioterapeuta para promoção do envelhecimento ativo e qual a melhor estratégia nesse processo. 
Após a redemocratização social e a incorporação da Constituição de 1988, algumas leis têm sido implantadas com o compromisso nacional de firmar ações de cunho constitucional e democrático de tratamento dos cidadãos idosos, sem distinções fundadas em preferências de gerações etárias, fortalecendo o respeito a todos os homens e mulheres ${ }^{10}$.

A Constituição Federal de 1988, no que se refere ao idoso, confere a irredutibilidade dos salários de aposentadoria e pensões, o amparo pelos filhos, a gratuidade nos transportes coletivos e o benefício de um salário mínimo àqueles sem condições de sustento ${ }^{5}$ e assegura, por meio do art. 230 , seus direitos ${ }^{11}$.

Em resposta ao crescimento da população idosa, o País tem estabelecido políticas públicas com o intuito de enfrentar as questões relacionadas com a saúde e com o bem-estar ${ }^{9}$, entre elas, a Política Nacional do Idoso (PNI) que foi aprovada em 1994, a Política Nacional de Saúde do Idoso (PNSI) implementada em 1999 e atualizada em 2006 para Política Nacional da Saúde da Pessoa Idosa (PNSPI) e o Estatuto do Idoso. Além disso, para enfrentamento dessas questões, o Brasil está de acordo com Plano de Ação Internacional para o Envelhecimento, de 2002. Ademais, a saúde do idoso é pauta importante de instrumentos que organizam o Sistema Único de Saúde (SUS) como o Pacto pela Vida (2006).

A Lei $n^{\circ} 8.842$, de 4 de janeiro de 1994, sancionou a $\mathrm{PNI}^{12}$. Essa lei teve como objetivo assegurar direitos sociais que garantam a promoção da autonomia, a integração e a participação efetiva do idoso na sociedade, de modo a exercer sua cidadania. Além disso, ela define o idoso no Brasil como sendo aquele com 60 anos ou mais, diferentemente de países desenvolvidos, que consideram como idoso o indivíduo com 65 anos ou mais?.

A PNI assegura ao idoso todos os direitos de cidadania, com a família, a sociedade e o Estado como responsáveis em garantir sua participação na comunidade, defender sua dignidade, bem-estar e direito à vida. Considera que o processo de envelhecimento diz respeito à sociedade, $\mathrm{e}$ o idoso não deve sofrer discriminação de nenhuma natureza, bem como deve ser o principal agente e o destinatário das transformações indicadas pela própria política. Por fim, cabe aos poderes públicos e à sociedade a aplicação da lei, considerando as diferenças econômicas e sociais, além das regionais ${ }^{9}$.

A PNSI, criada por meio da Portaria $n^{\circ} 1.395 / 1999$, do Ministério da Saúde (MS), é originada de uma necessidade de ampliar a discussão acerca do idoso. A perda da capacidade funcional foi levantada como principal problema que os afeta, aspecto que é influenciado pelo estilo de vida e enfermidades. Desse modo, a PNSI busca a promoção do envelhecimento saudável, a prevenção de doenças, a recuperação da saúde e a preservação/melhoria/ reabilitação da capacidade funcional dos idosos, com a finalidade de assegurar-lhes sua permanência no meio e sociedade em que vivem, desempenhando suas atividades de maneira independente ${ }^{13}$.

Outro marco no Brasil foi o Estatuto do Idoso de 2003, resultado dos movimentos de aposentados e pensionistas e influenciado pela II Assembleia Mundial das Nações Unidas, sediada em Madri ${ }^{5}$. 
Nessa Assembleia, foi elaborado o Plano de Ação Internacional para o Envelhecimento, fundamentado em três princípios básicos: 1) participação ativa dos idosos na sociedade, no desenvolvimento, na força de trabalho e na erradicação da pobreza; 2) promoção da saúde e bem-estar na velhice; e 3) criação de um ambiente propício e favorável ao envelhecimento ${ }^{14}$.

Considerando os princípios firmados na II Assembleia Mundial das $\mathrm{Na}$ ções Unidas, o Brasil lançou, em 2006, o Pacto Pela Vida, que definiu, entre as prioridades, a saúde do idoso e a implantação da PNSPI, aprovada em outubro de 2006, como resultado da revisão e atualização da PNSI de 1999 e com finalidade primordial: a recuperação, a manutenção e a promoção da autonomia e da independência da pessoa idosa, direcionando medidas coletivas e individuais em consonância com os princípios e diretrizes do SUS ${ }^{15}$.

Dentre as diretrizes norteadoras, destacam-se a Promoção do Envelhecimento Ativo e Saudável e a Atenção Integral e Integrada à saúde da Pessoa Idosa ${ }^{16}$. Nesse contexto, com o objetivo oferecer suporte à população sobre promoção da saúde, a Secretaria de Vigilância em Saúde produziu o documento "Envelhecimento Ativo: uma política de Saúde", elaborado com base no documento da Organização Mundial da Saúde (OMS) "Envelhecimento Saudável - Uma Política de Saúde" elaborado pela Unidade ${ }^{3}$.

Essas políticas possuem diretrizes, metas e objetivos em comum e as separam em seis áreas temáticas: acesso aos serviços de saúde; prevenção e manutenção da capacidade funcional; violência e maus tratos; moradia adequada; participação na comunidade; e assistência social.
Além disso, os autores apontam que as principais preocupações dos documentos estão relacionadas com o acesso aos serviços de saúde e com a prevenção e manutenção da capacidade funcional desses idosos ${ }^{4}$.

Durante o processo de envelhecimento, podem ocorrer alterações físicofuncionais que são características dessa etapa da vida, mas que estão propícias a mudar de acordo com as características individuais. Essas alterações predispõem o idoso a maior incidência das patologias e seus processos, além de maior vulnerabilidade, que podem comprometer a independência funcional ${ }^{17}$.

A Classificação de Incapacidade e Funcionalidade (CIF) aborda justamente a relação entre o adoecimento ou a limitação com as condições de saúde e com a percepção que o indivíduo tem de suas capacidades, levando em consideração as funções dos sistemas do corpo, as atividades diárias e participação social ${ }^{18}$. Sabe-se ainda que a funcionalidade dos idosos brasileiros é influenciada pelos fatores fisiológicos do envelhecimento e pelos determinantes sociais, como: renda, escolaridade, condições de saúde, ambiente físico e social que respondem a um gradiente geográfico de diferentes formas ${ }^{17}$.

O movimento é um item avaliado pela CIF. Um indivíduo com restrição de movimento sofre alterações fisiológicas, como enrijecimento da musculatura, fraqueza, osteoporose, alterações das características morfológicas, bioquímicas e biomecânicas. Essa condição pode repercutir em outras dimensões da vida, como psicológica, desenvolvendo ansiedade, apatia, depressão e/ou isolamento social ${ }^{19}$. 
Alguns hábitos podem se relacionar com a condição descrita acima, como o comportamento sedentário, caracterizado pela adoção de atividades que são realizadas na posição deitada ou sentada, que não ultrapassam o gasto energético acima dos níveis de repouso, levando ao desenvolvimento de doenças cardiovasculares, obesidade, diabetes e câncer. No dia a adia, tem-se adotado cada vez mais atividades com essas características, como andar de carro ou ônibus, assistir televisão, usar do computador, vídeo game, controle remoto, entre outras ${ }^{20}$.

Considerando a importância da realização das atividades físicas, preservar a funcionalidade no processo de envelhecimento tem sido relatado como um indicador de saúde, que interage com diversas funções, entre elas, a capacidade física e psicocognitiva, que sustentam a realização das atividades cotidianas e a participação social e comunitária ${ }^{19}$. Além disso, ela está diretamente associada à qualidade de vida dos idosos, já que está relacionada coma sua inserção no meio social ${ }^{21}$.

Dito isso, a adoção ao estilo de vida fisicamente ativo deve ser estimulada com o intuito de prevenir o desenvolvimento de doenças e a promoção da saúde do idoso.

\section{SAÚDE DO IDOSO E ATEN- ÇÃO PRIMÁRIA À SAÚDE}

O Envelhecimento Ativo foi definido pela OMS, em 2005, como: "processo de otimização das oportunidades de saúde, participação e segurança, com o objetivo de melhorar a qualidade de vida à medida que as pessoas ficam mais velhas"3.
Essa definição associa o envelhecer com o favorecimento de oportunidades para que os indivíduos tenham controle da sua própria saúde, por meio de escolhas relacionadas com estilo de vida saudável e, consequentemente, melhora da qualidade de vida ${ }^{22}$.

A OMS ainda aponta que, se as políticas públicas em saúde, o mercado de trabalho, emprego e educação apoiarem o envelhecimento ativo, espera-se menos deficiências associadas às DCNT, mais idosos participando ativamente nos aspectos sociais, culturais, econômicos e políticos da sociedade, além de menos gastos com tratamentos médicos e serviços de assistência médica. Ressalta também que isso depende de uma diversidade de fatores determinantes, como: pessoais, sociais, econômicos, comportamentais, ambiente físico, serviços sociais e de saúde, além de gênero e cultura ${ }^{3}$.

Em 2013, com o objetivo de conhecer o perfil da saúde da população brasileira, o MS, em conjunto com o Instituto Brasileiro de Geografia e Estatística, realizou a Pesquisa Nacional de Saúde (PNS) que levantou informações relativas ao estilo de vida da população. Os dados apontaram que $62,7 \%$ dos idosos brasileiros não praticam atividade física regularmente, mostrando que estes são os que apresentam maior prevalência de inatividade física. A pesquisa apontou também que $32,4 \%$ dos idosos assistem mais que três horas/dia de televisão, indicando que essa população é a que mais gasta tempo em comportamento sedentário ${ }^{2}$.

Diante disso, questiona-se a razão pela qual, mesmo com as políticas que estimulam e defendem estilos de vida saudáveis, esses dados retratam um panorama preocupante para com essa população. 
Nesse sentido, a Atenção Básica $(\mathrm{AB})$ ou Atenção Primária é porta de entrada da população, abrigando a Estratégia Saúde da Família (ESF), firmada e atualizada pela Portaria $n^{\circ} 2.436$, de 21 de setembro de 2017, prioridade para expansão e consolidação dessa política. De acordo com a Política Nacional de Atenção Básica (PNAB,) uma das atribuições das ESF é adscrever os usuários, o qual diz respeito à vinculação dos usuários ou famílias à unidade que será referência de atendimento às suas necessidades ${ }^{23}$.

Esse processo auxilia na construção de vínculos entre os profissionais das ESF e a população adscrita, favorecendo a criação de laços de compromisso e corresponsabilidade do cuidado por meio de uma logística de continuidade e longitudinalidade das ações em saúde. Além disso, o vínculo orienta ações da ESF, que se torna responsável pelo monitoramento e intervenção sobre os fatores de risco aos quais a população está exposta, prestando uma assistência integral permanente e de qualidade ${ }^{24}$.

Foi nesse panorama que surgiu a ESF, considerando a dinâmica de território, como uma potencialidade para identificar as necessidades, fomentar as ações de promoção da saúde ${ }^{25}$, almejando melhorias na qualidade de vida da população, por meio da promoção do envelhecimento saudável ${ }^{26}$. Por essa razão, esta consiste em um espaço favorecido para atenção integral à saúde do idoso, já que essa proximidade com a comunidade favorece a atuação no contexto vivenciado por este no seio familiar ${ }^{27}$.
No entanto, em 2013 , apenas 56,3\% dos idosos estavam adscritos na $\mathrm{ESF}^{2}$.

Outra estratégia que surge para fortalecer a $\mathrm{AB}$ são os Núcleos Ampliados de Saúde da Família e Atenção Básica (Nasf-AB), criados com o intuito de ampliar o escopo das ações por meio do compartilhamento de saberes, expandindo a resolutividade clínica das equipes. Os Nasf são equipes multiprofissionais, que devem atuar de maneira integrada e apoiando os profissionais das equipes de Saúde da Família e das equipes de AB. O fisioterapeuta é um dos profissionais atuantes no Nasf, que, com os demais profissionais do núcleo, tem como objetivo qualificar e dar suporte ao trabalho desenvolvido pelas equipes de Saúde da Família ${ }^{25}$.

\section{FISIOTERAPIA NA SAÚDE DO IDOSO}

A fisioterapia surgiu em um contexto histórico de atuação nos processos de reabilitação, visando à recuperação da função de habilidades perdidas e atuando, quase exclusivamente, no controle dos danos de determinadas doenças. A fisioterapia reabilitadora é destinada à cura de determinadas doenças e/ou à reabilitação de suas sequelas e complicações. Nesse caso, o objetivo da intervenção é o sujeito individualizado, e, às vezes, apenas partes ou órgãos isolados, o que destaca apenas alguns espaços de atuação como hospitais e clínicas de reabilitação. No entanto, esses espaços restringem e condicionam a intervenção apenas ao nível de atenção terciá- 
ria, o que impõe à população grande carga de doenças e sequelas, que poderiam ser evitadas. Dessa maneira, diante dos novos desafios da sociedade brasileira, como o quadro epidemiológico, a transição demográfica e a organização dos sistemas de saúde, surge a necessidade de reestruturação do objetivo de intervenção da fisioterapia, aproximando-se da promoção da saúde e ampliando, assim, a atuação do fisioterapeuta ${ }^{28}$.

Expandindo o campo de atuação, o fisioterapeuta atuará tanto com os idosos com alguma comorbidades já instaladas como também com os que ainda não apresentam nenhum acometimento, de forma que ambos possam buscar o envelhecimento ativo. Além disso, deve-se aproveitar a oportunidade de atuar como atores na prevenção de agravos e na mudança de hábitos. Esse incentivo à mudança de hábitos constitui um conjunto de ações da $\mathrm{AB}$, que utiliza recursos de baixa densidade com o intuito de promover mudanças no estilo de vida.

As estratégias como a Educação em Saúde, por meio de trabalhos em grupo, como rodas de conversas, são ótimos exemplos. Esta permite que o idoso obtenha informações acerca de sua saúde individual, proporcionando que ele participe do processo, como corresponsável pela sua saúde, estimulando assim sua autonomia, qualidade de vida e, consequentemente, um envelhecimento ativo. No entanto, o trabalho em grupo não deve ser apenas para suprir demandas, mas sim ser um espaço estimulador de troca de experiências e de vínculo social.

Os grupos de exercícios físicos oferecidos pela $\mathrm{AB}$ e pelos demais equipamentos sociais dentro do território adscrito são importantes ferramentas para a criação de vínculo, troca de saberes e fortalecimento social, além de ser uma estratégia para promoção de saúde e momento oportuno para aplicação da educação em saúde, promovendo a autonomia desse indivíduo.

De acordo com a Resolução $\mathrm{n}^{\circ}$ 476, de 20 de dezembro de 2016, que reconhece a especialidade profissional de fisioterapia em Gerontologia, o fisioterapeuta com essa especialidade deve ser capaz, além de realizar consulta e diagnóstico fisioterapêutico/cinesiológico-funcional com ênfase na capacidade funcional, referente à autonomia e à independência das pessoas em processo de envelhecimento -, ser capaz também de planejar e executar ações de prevenção e redução de risco, medidas de promoção de saúde, manutenção da capacidade funcional, prevenção de doenças/agravos próprios do processo de envelhecimento; bem como de almejar o estado de máxima funcionalidade ${ }^{29}$.

\section{CONSIDERAÇÕES FINAIS}

O processo de envelhecimento é complexo e influenciado por fatores e determinantes que implicam a não adoção de hábitos saudáveis, que levam ao envelhecimento ativo. Sabe-se que a atuação do fisioterapeuta ainda encontra diversas barreiras no escopo de suas ações, sendo, muitas vezes, ainda visto como o profissional responsável pela reabilitação e designado a suprir somente as demandas dessa área. No entanto, deve-se buscar cada vez mais sua atuação incentivadora do envelhecimento ativo, promotora das condições para desenvolvimento de exercícios e atividades físicas e dos processos de educação em saúde 


\section{REFERÊNCIAS}

1. Carneiro LAF, Campino ACC, Leite F, Rodrigues CG, Santos GMM, Silva ARA. Envelhecimento Populacional e os desafios para o sistema de saúde Brasileiro [internet]. Instituto de Estudos de Saúde Suplementar - IESS, São Paulo; 2013 [acessado 2020 mar 3]. Disponível em: https://www.iess. org.br $/$ ? $\mathrm{p}=$ publicacoes\&id $=436 \& \mathrm{id}$ tipo $=3$.

2. Brasil. Instituto Brasileiro de Geografia e Estatística. Pesquisa Nacional por Amostra de Domicílios Contínua - PNAD Contínua [internet]. Brasília, 2013 [acessado 2020 mar 3]. Disponível em: http://www.governo.gov.ao/ download.aspx?id=1264\&tipo $=$ publicacao.

3. Organização Mundial da Saúde (OMS). Envelhecimento ativo: uma política de saúde [internet]. 2005 [acessado 2020 mar 3]. Disponível em: http://bvsms.saude.gov.br/bvs/publicacoes/envelhecimento_ativo.pdf.

4. Romero DE, Pires DC, Marques A, Muzy J. Diretrizes e indicadores de acompanhamento das políticas de proteção à saúde da pessoa idosa no Brasil. Rev Eletron Comun Inf Inov Saúde 2019;13(1):134-157.

5. Veras RP, Oliveira M. Envelhecer no Brasil: a construção de um modelo de cuidado. Ciênc saúde coletiva 2018; 23:1929-1936.

6. Alves LC, Leimann BCQ, Vasconcelos MEL, Carvalho MS, Vasconcelos AGG, Fonseca TCO, et al. A influência das doenças crônicas na capacidade funcional dos idosos do Município de São Paulo, Brasil. Cad Saúde Pública 2007; 23:1924-1930.

7. Renato V. Envelhecimento populacional contemporâneo: demandas, desafios e inovações. Rev Saúde Pública 2009; 43 (3): 548-554.
8. Maia FES, Moura ELR, Madeiros EC, Carvalho RRP, Silva SAL, Santos GR. A importância da inclusão do profissional fisioterapeuta. Rev Fac Ciênc Méd Sorocaba 2015; 17(3):110-115.

9. Fernandes MTO, Soares SM. O desenvolvimento de políticas públicas de atenção ao idoso no Brasil. Rev esc enferm USP 2012; 46(6):1494-1502.

10. Andrade LM, Sena ELS, Pinheiro GML, Meira EC, Lira LSSP. Políticas públicas para pessoas idosas no Brasil: uma revisão integrativa. Ciênc saúde coletiva 2013; 18(12):35433552.

11. Brasil. Constituição (1988) Constituição da República Federativa do Brasil. [internet] Brasília, DF: Senado Federal; 1988 [acessado 2020 mar 3]. Disponível em: https://www.senado.leg.br/atividade/const/con1988/ CON1988_05.10.1988/CON1988. asp.

12. Brasil. Política Nacional do Idoso. [internet]. Brasília; 1994 [acessado 2020 mar 3]. Disponível em: http://www. planalto.gov.br/ccivil_03/leis/18842. htm.

13. Silvestre JA, Costa Neto MM. Abordagem do idoso em programas de saúde da família. Cad Saúde Pública 2003; 19(3):839-847.

14. Organização das Nações Unidas. Plano de Ação Internacional para o Envelhecimento. [internet]. Brasília; 2003 [acessado 2020 mar 3]. Disponível em: http://www.observatorionacionaldoidoso.fiocruz.br/biblioteca/_ manual/5.pdf. 
15. Brasil. Ministério da Saúde. Portaria $\mathrm{n}^{\circ} 399$, de 22 de fevereiro de 2006. Divulga o Pacto pela Saúde 2006 Consolidação do SUS e aprova as Diretrizes Operacionais do Referido Pacto. [internet]. [Diário Oficial da União 2006 [acessado 2019 jun 13]; 23 fev. http://bvsms.saude. gov.br/bvs/saudelegis/gm/2006/ prt0399_22_02_2006.html.

16. Brasil. Departamento de Apoio à Gestão Descentralização. Colegiado de gestão regional na região de saúde intraestadual: orientações para organização e funcionamento. [internet]. Brasília, DF: Ministério da Saúde, Secretaria-Executiva, Departamento de Apoio à Gestão Descentralizada; 2009 [acessado 2020 mar 3]. Disponível em: http://bvsms.saude.gov.br/ bvs/publicacoes/pacto_saude_volume10.pdf.

17. Lopes GL, Santos MIPO. Funcionalidade de idosos cadastrados em uma unidade da Estratégia Saúde da Família segundo categorias da Classificação Internacional de Funcionalidade. Rev bras geriatr gerontol 2015; 18(1):71-83.

18. Farias N, Buchalla CM. A classificação internacional de funcionalidade, incapacidade e saúde da organização mundial da saúde: conceitos, usos e perspectivas. Rev bras epidemiol 2005; 8(2):187-193.

19. Boechat JCS, Castro Manhães F, Gama Filho RV, Istoé RSC. A síndrome do imobilismo e seus efeitos sobre o aparelho locomotor do idoso. InterScience Place 2012; 1(5):89-107.

20. Gomes dos Santos R, Medeiros JC, Schmitt BD, Meneguci J, Santos DAT, Damião $\mathrm{R}$, et al. Comportamento Sedentário em Idosos: uma Revisão Sistemática. Motri 2015; 11(3):171186.

21. Duarte LSS, Dutra CDT, Pires CAA, Assis FSJS, Silva DCB, Costa LS. Análise da capacidade funcional de idosos atendidos pela estratégia de saúde da família. Rev para med 2012; 26(4).
22. Campos ACV, Ferreira EF, Vargas AMD. Determinantes do envelhecimento ativo segundo a qualidade de vida e gênero. Ciênc saúde coletiva 2015; 20(7):2221-2237.

23. Brasil. Ministério da Saúde. Portaria ${ }^{\circ}$ 2.436, de 21 de setembro de 2017. Aprova a Política Nacional de Atenção Básica, estabelecendo a revisão de diretrizes para a organização da Atenção Básica, no âmbito do Sistema Único de Saúde (SUS). [internet]. Diário Oficial da União 2017 [acessado 2019 jun 13]; 22 set. Disponível em: http://bvsms.saude.gov.br/bvs/saudelegis/gm/2017/ prt2436_22_09_2017.html.

24. Prado NMBL, Santos AM. Promoção da saúde na Atenção Primária à Saúde: sistematização de desafios e estratégias intersetoriais. Saúde debate 2018; 42 (n.spe1): 379-395

25. Souza MC, Bonfim AS, Souza JN, Franco TB. Fisioterapia e Núcleo de Apoio à Saúde da Família: conhecimento, ferramentas e desafios. Mundo Saúde 2013; 37(2):176-184.

26. Motta LB, Aguiar AC, Caldas CP. Estratégia Saúde da Família e a atenção ao idoso: experiências em três municípios brasileiros. Cad Saúde Pública 2011; 27(4):779-786.

27. Oliveira JCA, Tavares DMS. Atenção ao idoso na estratégia de Saúde da Família: atuação do enfermeiro. Rev esc enferm. USP 2010; 44(3):774781.

28. Bispo Júnior JP. Fisioterapia e saúde coletiva: desafios e novas responsabilidades profissionais. Ciênc saúde coletiva 2010; 15(Supl.1):1627-1636

29. Conselho Federal de Fisioterapia e Terapia Ocupacional. Resolução $\mathrm{n}^{\circ}$ 476, de 20 de dezembro de 2016. Reconhece e Disciplina a Especialidade Profissional de Fisioterapia em Gerontologia e dá outras providências. [internet] Diário Oficial da União 2017 [acessado 2020 mar 3]; 19 jan. Aprovado: 04/03/2020
Disponível em: https://www.coffito. gov.br/nsite/?p=6303. 